\title{
Uniting the Prolog Community
}

\author{
Tom Schrijvers` and Bart Demoen \\ Department of Computer Science, K.U.Leuven, Belgium
}

In his article $A$ Wake-Up Call for the Logic Programming Community, published in the December 2007 issue of the ALP Newsletter, the first author raised concerns about the viability of the Prolog programming language in the near future. The article had a pessimistic undertone, but expressed the hope that the current evolution can be reversed by uniting the Prolog community. Now, almost a year later, it is time for more optimistic news and we are happy that the ALP - through the ICLP 2008 program chairs - has given us the opportunity to re-iterate the arguments, present the recent positive developments and involve the whole community.

The concerns raised in the ALP Newsletter have been known for years, but it takes repeated raising of voices to make the community more aware of the issues: one big problem is that the Prolog community has been too fragmented. This is true at several levels: at the system side one notes that there are too many incompatible Prolog systems around, their structure and libraries are different, their implementation technology is very diverse, and their aims are difficult to reconcile (research or industrial deployment). In short: unity lacks, there is too little community feeling. Instead, competition rules, and it is no healthy competition.

At the user base we see a wide range, from enthusiastic beginning Prolog programmers who need basic books and tutorials, students who are forced to learn Prolog for their courses, and professionals making money because they master the most intricate aspects of Prolog programming. Despite some thriving system specific user mailing lists, there is a lack of community feeling. The silence on comp.lang.prolog is a witness for that.

And there is the ISO problem: while the expectations were high that a standard would increase the acceptance of Prolog in wider circles, and that it would become effectively more easy to run the same Prolog program under several engines, the reality is different. Despite many (also recent) efforts, the ISO committee seems unable to make substantial progress on the core standard, let alone to impose the modules standard.

As for applications ... each system's maintainer has a few private success stories, but who remembers The Prolog 1000 Database? Don't we want it back?

Some of these issues can be solved only by (re-)uniting the community at all levels, for others more drastic actions are needed. But let us describe what has happened already, and why this rekindles our faith in a prosperous future for Prolog.

* Tom Schrijvers is a post-doctoral researcher of the Fund for Scientific Research Flanders. 
About a year ago, Jan Wielemaker and Vitor Santos Costa - the main drives behind SWI-Prolog and Yap - started talking concretely about letting their systems evolve towards each other. One important issue was the library structure and its contents. Jan and Vitor had been stealing each other's code already, but more was needed. Since then, real progress on the libraries has been made, and how this was achieved gradually is reported on in the event at ICLP 2008. Quite importantly is here also that the operator import/(re-)export facilities were synchronized. Other issues are being talked about and will be resolved, sometimes on a per-need basis: both systems support threads and it would be nice if they adhered to a common standard. Maybe ISO could play a role here, but it should be quick! Both systems have a different C-interface: Yap already can emulate the SWI-Prolog C-interface, but more commonality is the plan. The module system is another hot potato: neither of the systems is inclined to implement ISO part II on Modules, but the current differences between them hinder other common functionality related to libraries and term expansion. SWI-Prolog has (once more) plans to incorporate tabling. And very recently, the first (big) steps were set towards a common (optional) type system (see somewhere else in these proceedings).

The importance of this collaboration is less in what has been achieved until now, than in the fact that it shows the will and commitment to defragment the Prolog systems landscape, and to unite two popular systems. Such collaboration enhances the enthusiasm of implementors, contributors and users alike. From now on, a library contributed to Yap, is useful to the user community of two Prolog systems, not one. From now on, the application written for SWI-Prolog will run on Yap, and while developing it might have been easier in the former, running it might be faster in the latter: the application writer really benefits from the larger compatibility.

Sure, we are being carried away a bit here, but that is the result of being involved in joining forces: optimism!

The ALP Newsletter article mentioned earlier set a challenge to the Prolog systems. The challenge was for two Prolog systems to team up and start collaborating on mutual compatibility and shared libraries. That challenge has been met: the two cooperating systems present themselves at ICLP 2008.

The other challenge is to the Prolog community as a whole: let's work together on a more collaborative Prolog programming environment and actively support the effort. There are many ways to do so: by conducting research on these systems and citing them, by contributing library code and improving documentation, by teaching our students to use these systems and giving them assignments for writing libraries and tools. And very importantly, more Prolog systems must enter the collaboration: they are very welcome! That is the way to make our community grow, to attract new people and raise business interest.

Acknowledgements The first author is grateful for all the positive feedback from the community on his ALP Newsletter article. 